\title{
Past, present and future
}

\author{
Jon Karlsson $\cdot$ Roland Becker $\cdot$ Neil P. Thomas
}

Published online: 7 November 2012

(c) Springer-Verlag Berlin Heidelberg 2012

We would like to start by thanking everyone who has been involved in the journal during the year, especially the reviewers who are listed here. Reviewing a manuscript is time-consuming and unpaid, and often reviewers are bombarded with requests to review simultaneously from several journals. We are very much aware of this, so we try and limit the number of reviews as much as possible. But we are also aware that we lean heavily on the same reviewers over and over again due to their excellence. In other words, 'the only reward for doing good work is more work!...' As Editors, we are constantly looking for new reviewers to reduce and redistribute the workload. In fact, we have been successful in this task, and currently, there are more than 500 active reviewers on our list. We try hard to use reviewers in a sensible manner, not to overload them and only give them papers that are closely related to their field of expertise. If we do not succeed all the time, it is not for want of trying. We should like to thank all the reviewers for their efforts during 2012. Your work is invaluable, and without you, the journal would simply not survive.

At the ESSKA congress in Geneva, we selected for the first time the 'Outstanding reviewers of the year'. This is something that will be repeated. For the year 2011, Olaf Lorbach (Germany) and Stavros Ristanis (Greece) were

J. Karlsson $(\bowtie)$

Department of Orthopaedics, Sahlgrenska University Hospital, Mölndal, Sweden

e-mail: jon.kssta@gmail.com

R. Becker

Department of Orthopaedic and Traumatology, City Hospital Brandenburg, Hochstrasse 29, 14770 Brandenburg/Havel, Germany

\section{N. P. Thomas}

Winchester, UK nominated. We are now able to announce the 'Outstanding reviewers of 2012' - two young, bright people both originating from the Netherlands, but currently in United States. They are Carola van Eck (Pittsburg, PA) and Coen Wijdicks (Vail, CO). Not only are their reviews of rigorous scientific quality, but they deliver punctually-a dream for Editors. Congratulations to you both.

This year has been good for the journal in many ways. The Editorial Office is now very well established and has recently been strengthened with an additional part-time editorial secretary. The Editorial Office is now better co-ordinated with the ESSKA office in Luxembourg, under the leadership of Elodie Reyter, Editorial Co-ordinator. Our new website has been launched (www.kssta.org), and we now have several hundred friends on our Facebook page. We feel that all these activities are rewarding both for authors and for readers of the journal, and we will do our best to continue the momentum in this medium.

We are encouraged that KSSTA's Impact Factor has now climbed beyond the 'magic number' of 2.0. The Impact Factor for 2011 was released in June and was 2.209. This places the journal in an enviably high position in the categories of both sports medicine and orthopaedics. However, further improvements are both mandatory and achievable, and we will all need to work hard to deliver this. We need your help with the best papers and the best reviews.

At the ESSKA meeting in Geneva, we celebrated the journal's 20th anniversary during a well-attended ceremony. The best scientific papers of 2010-2011 were selected, with Pau Golanó (Spain) and co-workers as winners, Markus Waldén (Sweden) and co-workers as runners-up and Volker Musahl (USA) and co-workers and Guillaume Demey (France) and co-workers in joint third place. We will repeat this at the ESSKA meeting in Amsterdam with the best papers of 2012-2013. 
Now, what lies in future? Probably, the journal has more or less reached its maximum size with 2,600 pages this year and a planned 2,800 for 2013. Submissions have exceeded 1,000 for the first time, and whilst this is an interesting landmark, its significance can be debated. We prefer to receive fewer papers of higher quality. But what is most important is the trend that we have seen with a larger number of better papers and less poorer ones.

We plan some changes to the Editorial Board, there will be expansion, and the new additions will be introduced in the January 2013 issue. They include Michael Hantes,
Hélder Pereira, Oliver Kessler, Michael Bohnsack, Peter Balcarek, Kristian Samuelsson, Mustafa Karahan, Eduard Alentorn-Geli, Géza Pap, Martin Lind, Jens Agneskirschner, Eva Zeizig, Peter Angele, Kristín Briem and Christoph Becher. All have done outstanding work as reviewers during the last few years.

Finally, we would like to thank René Verdonk for his major contribution to the journal. René has now retired from his position as Editor-in-Chief, but will still assist on special projects, as Senior Editor. Thank you René for everything.

\section{2 reviewers}

Henrik, Aagaard, Denmark

Paul, Ackermann, Sweden

Nobuo, Adachi, Japan

Paolo, Adravanti, Italy

Jens, Agneskirchner, Germany

Mattias, Ahldén, Sweden

Eduard, Alentorn-Geli, Spain

Alberto, Aliprandi, Italy

Fredrik, Almqvist, Belgium

Bogdan, Ambrozic, Slovenia

Annunziato, Amendola, USA

Andrew, Amis, UK

Allen, Anderson, USA

Jack, Andrish, USA

Peter, Angele, Germany

Pierluigi, Antinolfi, Italy

Yoshimitsu, Aoki, Japan

Elizabeth, Arendt, USA

Jean Noel, Argenson, France

Toni, Arndt, Sweden

Markus, Arnold, Switzerland

Asbjørn, Årøen, Norway

Carl, Askling, Sweden

Jesper, Augustsson, Sweden

Klaus, Bak, Denmark

Peter, Balcarek, Germany

Andrea, Baldini, Italy

Adad, Baranto, Sweden

John, Bartlett, Australia

Thomas, Bauer, France

Philippe, Beaufils, France

Christoph, Becher, Germany

Johannes, Beckmann, Germany

Franco, Benazzo, Italy

Stephen, Bendall, UK

Anders, Bengtsson, Sweden

Simone, Bignozzi, Italy

Michel, Bonnin, France
Ulrich, Bosch, Germany

Sveinbjörn, Brandsson, Iceland

Kristín, Briem, Iceland

Karen, Briggs, USA

Torkel, Brismar, Sweden

Peter, Brucker, Germany

Danilo, Bruni, Italy

Nicolaas, Budhiparama, Indonesia

Anthony, Bull, UK

Gianluca, Camillieri, Italy

Mike, Carmont, UK

Antonio, Cartucho, Portugal

Alessandro, Castagna, Italy

Fabio, Catani, Italy

ChengKung, Cheng, Taiwan,

Republic of China

Pascal, Christel, Saudi Arabia

Svend-Erik, Christiansen, Denmark

Mark, Clatworthy, New Zealand

Ramon, Cugat, Spain

Diane, Dahm, USA

Tore, Dalen, Sweden

Patrik, Danielson, Sweden

Ricardo, de Casas, Spain

Maarten, de Waal Malefijt,

Netherlands

Lieven, De Wilde, Belgium

Kamal, Deep, UK

Koen, Defoort, Netherlands

Louis, DeFrate, USA

Masataka, Deie, Japan

David, Dejour, France

Guillaume, Demey, France

Patrick, Djian, France

Simon, Donell, UK

Brian, Donley, USA

Mahmut, Doral, Turkey

Matej, Drobnic, Slovenia
Jon, Drogset, Norway

Irem, Duzgun, Turkey

Fredrik, Einarsson, Sweden

Lars, Ejerhed, Sweden

Jan, Ekstrand, Sweden

Scott, Ellis, USA

Lars, Engebretsen, Norway

Karl, Eriksson, Sweden

Bengt, Eriksson, Sweden

Jaroslaw, Fabis, Poland

Jack, Farr, USA

Peter, Faun $\varnothing$, Denmark

Julian, Feller, Australia

Julian, Fernandez-Gonzalez, Spain

Andrea, Ferretti, Italy

Daniel, Fong, China

Fernando, Fonseca, Portugal

Francisco, Forriol, Spain

Magnus, Forssblad, Sweden

Alois, Franz, Germany

Niklaus, Friederich, Switzerland

Richard, Frobell, Sweden

Freddie, Fu, USA

Susanne, Fuchs-Winkelmann,

Germany

John, Fulkerson, USA

John, Galbraith, Ireland

João, Gamelas, Portugal

Raffaele, Garofalo, Italy

J Robert, Giffin, Canada

Thomas, Gill, USA

Francesco, Giron, Italy

John, Gliatis, Greece

Alberto, Gobbi, Italy

Alli, Gokeler, Netherlands

Andreas, Gomoll, USA

Simon, Gortz, USA

Hans, Granhed, Sweden 
Karin, Grävare Silbernagel, USA

Paolo, Grossi, Italy

Martin, Hägglund, Sweden

Andreas, Halder, Germany

Kjartan, Halvorsen, Sweden

Frank, Handelberg, Belgium

Milan, Handl, Czech Republic

Laslo, Hangody, Hungary

Michael, Hantes, Greece

Arsi, Harilainen, Finland

Koji, Hattori, Japan

Joerg, Hausdorf, Germany

Petra, Heesterbeek, Netherlands

Annette, Heijne, Sweden

Philipp, Henle, Switzerland

Pedro, Hinarejos, Spain

Stefan, Hinterwimmer, Germany

Michael, Hirschmann, Switzerland

Lorenz, Hofbauer, Germany

Siegfried, Hofmann, Austria

Jürgen, Höher, Germany

Stephen, Howell, USA

Christohp, Hulet, France

Christoph, Hurschler, Germany

Andreas, Imhoff, Germany

Sheila, Ingham, Brazil

Michael, Iosifidis, Greece

Takanori, Iriuchishima, Japan

Alan, Ivkovic, Croatia

Michael, Jagodzinski, Germany

Karl-Åke, Jansson, Sweden

Timo, Jarvela, Finland

Mislav, Jelic, Croatia

Jean-Yves, Jenny, France

Joerg, Jerosch, Germany

Peter, Kälebo, Sweden

Mustafa, Karahan, Turkey

Philip, Kasten, Germany

Defne, Kaya, Turkey

Daniel, Kendoff, Germany

Gino, Kerkhoffs, Netherlands

Oliver, Kessler, Switzerland

Wasim, Khan, UK

Wolfgang, Klauser, Germany

Burt, Klos, Netherlands

Karsten, Knobloch, Germany

Gunnar, Knutsen, Norway

Koichi, Kobayashi, Japan

Jason, Koh, USA

Dieter, Kohn, Germany

Elizaveta, Kon, Italy

Eiji, Kondo, Japan
Sebastian, Kopf, Germany

Nanne, Kort, Netherlands

Ioannis, Kostogiannis, Sweden

Ladislav, Kovacic, Slovenia

Marius, Kraenzlin, Switzerland

Thomas, Krüger, Germany

Joanna, Kvist, Sweden

Robert, LaPrade, USA

Chris, Larson, USA

Constance, Lebrun, Canada

Sang Hak, Lee, Republic of Korea

Alberto, Lemos, Portugal

Pisit, Lertwanich, Thailand

Bruce, Levy, USA

Ofer, Levy, UK

Guoan, Li, USA

Dennis, Liem, Germany

Martin, Lind, Denmark

Anders, Lindahl, Sweden

Sverre, Löken, Norway

Umile Giuseppe, Longo, Italy

Olaf, Lorbach, Germany

Mari, Lundberg, Sweden

Sebastien, Lustig, Australia

Jack, Lysholm, Sweden

C. Benjamin, Ma, USA

Peter, Macdonald, Canada

Henning, Madry, Germany

Nicola, Maffulli, UK

Martin, Majewski, Switzerland

David, Mandeville, USA

Maurillio, Marcacci, Italy

Fabrizio, Margheritini, Italy

Stefan, Marlovits, Austria

Hal, Martin, USA

Inigo, Martinez, Norway

Cesar, Martins, Brazil

Nimalan, Maruthainar, UK

Robert, Marx, USA

Dimitrios, Mastrokalos, Greece

Tomoyuki, Matsumoto, Japan

Georg, Matziolis, Germany

Hermann-Otto, Mayr, Germany

Ingmar, Meinecke, Germany

Khaled, Meknas, Norway

Rupert, Meller, Germany

Jacques, Menetrey, Switzerland

Numa, Mercier, France

Duncan, Meuffels, Netherlands

Giuseppe, Milano, Italy

Maarten H, Moen, Netherlands

Juergen, Mollenhauer, Germany
Ferran, Montserrat, Spain

Nuno, Moura, Portugal

Thomas, Muellner, Austria

Takeshi, Muneta, Japan

Volker, Musahl, USA

Peter, Myers, Australia

Florian, Naal, Switzerland

Norimasa, Nakamura, Japan

Wolfgang, Nebelung, Germany

Katarina, Nilsson-Helander, Sweden

John, Nyland, USA

Mitsuo, Ochi, Japan

Shawn, O’Driscoll, USA

Padhraig F, O'Loughlin, USA

Patrick, Orth, Germany

Sven, Ostermeier, Germany

Géza, Pap, Germany

Dietrich, Pape, Luxembourg

David, Parker, Australia

Sebastien, Parratte, France

Cecilia, Pascual-Garrido, USA

Thilo, Patzer, Germany

Christopher, Pearce, Singapore

Andrew, Pearle, USA

Luigi, Pederzini, Italy

Giuseppe, Peretti, Italy

Carsten, Perka, Germany

Frank, Petrigliano, USA

Tilman, Pfitzner, Germany

Marc, Philippon, USA

Anand, Pillai, UK

Kevin, Plancher, USA

Chadwick, Prodromos, USA

Nicolas, Pujol, France

Hans, Rahme, Sweden

Ashok, Rajgopal, India

Pietro, Randelli, Italy

Filippo, Randelli, Italy

Per, Renström, Sweden

Stavros, Ristanis, Australia

Henri, Robert, France

Sally, Roberts, UK

Christer, Rolf, Sweden

Harald, Roos, Sweden

Roberto, Rossi, Italy

Jan Harald, Røtterud, Norway

Oliver, Rühmann, Germany

Miguel, Ruiz Iban, Spain

Junnosuke, Ryu, Japan

Tönu, Saartok, Sweden

Greg, Saboeiro, USA

Björn, Salomonsson, Sweden 
José Filipe, Salreta, Portugal

Massimiliano, Salvi, Italy

Kristian, Samuelsson, Sweden

Dominique, Saragaglia, France

Daniel, Saris, Netherlands

Martin, Sauerschnig, Germany

Markus, Scheibel, Germany

Alfredo, Schiavone Panni, Italy

Philip, Schoettle, Germany

Roberto, Seijas, Spain

Romain, Seil, Luxembourg

Ninni, Sernert, Sweden

Elvire, Servien, France

Konsei, Shino, Japan

Luminita, Simion, Germany

Konrad, Slynarski, Poland

Tomislav, Smoljanovic, Croatia

Satoshi, Soen, Japan

Francesc, Soler, Spain

David, Sonnabend, Australia

Gunter, Spahn, Germany

Kurt, Spindler, USA

Christian, Stärke, Germany

Nick, Stergiou, USA
Robin, Strachan, UK

Klemen, Strazar, Slovenia

Michael, Stuart, USA

Jorma, Styf, Sweden

Eduardo, Suero, USA

Daisuke, Suzuki, Japan

Imre, Szerb, Hungary

Koji, Takayama, Japan

Scott, Tashman, USA

Magnus, Tengvar, Sweden

Roland, Thomee, Sweden

Brady, Tripp, USA

Elias, Tsepis, Greece

Michel PJ, van den Bekerom, Netherlands

Henk, Van der Hoeven, Netherlands

Carola, van Eck, USA

Nicolien, Van Giffen, Luxembourg

Ronald, van Heerwaarden,

Netherlands

Corné, Van Loon, Netherlands

Hilde, Vandenneucker, Belgium

Alberto, Ventura, Italy

Peter, Verdonk, Belgium
Jason, Vescovi, Canada

Jan, Victor, Belgium

Luis, Videla, Chile

Mihai H, Vioreanu, Ireland

Piero, Volpi, Italy

Fabian, von Knoch, Switzerland

Dharmesh, Vyas, USA

Ola, Wahlström, Sweden

Lars, Weidenhielm, Sweden

Thomas, Wickiewicz, USA

Wojciech, Widuchowski, Poland

Coen, Wijdicks, USA

Jaap, Willems, Netherlands

Philippe, Wilmes, France

Timothy, Wilton, UK

Brian, Wolf, USA

Barbara, Wondrasch, Austria

Kazunori, Yasuda, Japan

Yi-Meng, Yen, USA

Shinichi, Yoshiya, Japan

James, Zachazewski, USA

Eva, Zeisig, Sweden

Jin, Zhong-Min, UK 